\title{
Histoire et histoires. Politique et poétique des récits miniers dans le Pacifique Sud
}

History and stories. The politics and poetics of mining narratives in the South Pacific

Eddy Banaré et Pierre-Yves Le Meur

\section{CpenEdition}

\section{Journals}

Édition électronique

URL : http://journals.openedition.org/jso/7033

DOI : $10.4000 /$ jso.7033

ISSN : $1760-7256$

Éditeur

Société des océanistes

Édition imprimée

Date de publication : 15 décembre 2014

Pagination : 5-22

ISBN : 978-2-85430-118-2

ISSN : 0300-953x

\section{Référence électronique}

Eddy Banaré et Pierre-Yves Le Meur, « Histoire et histoires. Politique et poétique des récits miniers dans le Pacifique Sud ", Journal de la Société des Océanistes [En ligne], 138-139 | 2014, mis en ligne le 15 décembre 2014, consulté le 23 septembre 2020. URL : http://journals.openedition.org/jso/7033 ; DOI : https://doi.org/10.4000/jso.7033 


\title{
Introduction : Histoire et histoires. Politique et poétique des récits miniers dans le Pacifique Sud
}

par

\author{
Pierre-Yves LE MEUR ${ }^{*}$ et Eddy BANARÉ ${ }^{* *}$
}

\begin{abstract}
"Contemporary literary theory is much too useful to let it be limited to the domains of critique, reflexivity, and value pluralism alone." (Roe, 1994 : 10)
\end{abstract}

L'objectif de ce dossier est de mettre en lumière la variété tout comme les points de convergence des questions posées par l'exploitation minière dans le Pacifique, et ce au prisme de la mise en récit de cette activité : comment la mine est-elle racontée ? Par qui, dans quels contextes, à quelles fins et avec quels effets? Les articles rassemblés ici traitent de ce thème en proposant des analyses et des contextualisations de textes, situations et héritages historiques, ainsi que de productions artistiques qui sont autant de formes narratives portant sur la mine dans la Pacifique ${ }^{1}$. Il s'agit ainsi de donner à voir la variété des expériences humaines de la mine dans cette région à travers la multiplicité des narrations évoquant les discours, les pratiques et les représentations de cet objet et enjeu si particulier.

C'est justement par l'objet que nous entamerons cette introduction, à savoir la mine dans sa matérialité, à la fois comme enclave physique au sein de laquelle s'effectue un procès de travail spécifique (Sidaway, 2007), et dans ses "débordements " (Letté, 2009) extérieurs, ses impacts sur la société qui l'environne. Cette première section nous permettra de jeter les bases de la réflexion sur la manière dont se sont construites des formes narratives portant de manière très variées sur cet objet et/ou ses effets. La seconde section balisera le champ sémantique défini par la notion de récit en recourant à la théorie littéraire. Nous procéderons dans la troisième section à un élargissement de la problématique en direction des usages philosophiques, politiques et anthropologiques de la notion de récit - en particulier les récits sans auteur clairement défini, mais pas sans narrateurs : grand récits, métarécits ou récits de politique publique - avant de revenir sur la question du narrateur dans les formes de « mise en intrigue " de la mine dans le Pacifique.

La mise en intrigue bâtit sur différents modes, comme nous le verrons, un lien entre temps vécu et temps raconté, entre narration et action, dans la mesure où, "par la vertu de l'intrigue, des buts, des causes, des hasards sont rassemblés sous l'unité temporelle d'une action totale et complète" (Ricour, 1983: 9). Or, remarque nécessaire, si ce dossier présente un échantillon

1. Plusieurs textes (Banaré, Bencivengo, Chaloping-March, Edwards, Leach, Pollock) sont issus de présentations faites dans le cadre du colloque Exploitation et politique minières dans le Pacifique : histoire, enjeux et perspectives (IRD-CPSIAC-CORAIL-CIRAD-UNC-CNRT " Nickel et son environnement ») qui s'est tenu à Nouméa du 21 au 25 novembre 2011. Ils ont été retravaillés pour aboutir aux versions finales publiées ici. D’autres présentations ont alimenté un ouvrage à paraître à l'ANU (voir Filer et al., à paraître). Nous remercions ici chaleureusement pour leurs lectures et commentaires Michel Jébrak, Dan Jorgensen, Claire Levacher, ainsi que le comité de rédaction du JSO et le lecteur anonyme.

*Anthropologue, Institut de recherche pour le développement (IRD, Nouméa), pierre-yves.lemeur@ird.fr

"* Docteur en littérature, chercheur associé au Centre des nouvelles études du Pacifique (cNep, Université de la NouvelleCalédonie), eddy.banare@gmail.com 
significatif d'une diversité de récits miniers, il n'épuise pas le sujet et n'est pas complètement représentatif de son ampleur et de sa variété. Les "récits sans auteur" que sont les récits de la colonisation, du développement ou de la malédiction des ressources traversent la plupart de ces textes, dont certains font de cette entrée la problématique centrale (Bencivengo, ChalopingMarch, Pollock par exemple). Les approches plus ethnographiques varient entre, d'une part, l'établissement d'une correspondance entre type de récits et groupe d'acteurs dans le cadre d'une analyse en termes d'arène minière (en particulier Burton) et, d'autre part, un accent mis sur la variété des récits et des pratiques développés par les populations touchées par la mine (en particulier Bacalzo et al., Jorgensen, Leach). Selon les articles, la notion de récit, telle que nous allons en développer les caractéristiques et l'intérêt dans cette introduction, est fortement mise en œuvre (Banaré pour le champ littéraire, Giulani de manière plus métaphorique pour le champ artistique), ou alors elle apparait comme une trame analytique nous permettant de mettre en perspective des études de cas à caractère historique (Edwards, Nanau).

\section{La mine dans le Pacifique : récit et matérialité}

L'exploitation minière dans le Pacifique se caractérise par sa diversité, plus précisément par son inscription dans les trajectoires historiques spécifiques de territoires et pays aux destins variés. Enjeu économique, social et environnemental souvent majeur, la mine a traversé l'époque coloniale et continue d'imprimer sa marque sur les pays et territoires d'une région caractérisée par une transition tardive vers la décolonisation. Ses implications politiques et ses impacts socio-environnementaux sont quant à eux extrêmement divers. Selon les lieux du Pacifique où elle s'est déployée, l'activité minière a pu être génératrice de désastres environnementaux ou bien le déclencheur de processus de développement économique, ou encore un facteur de troubles politiques (voir Ballard and Banks, 2003; Bebbington et al., 2008). Les années 1990 ont vu non pas l'émergence mais la forte médiatisation de dommages massifs causés par l'activité minière. Pour la zone AsiePacifique, deux cas sont emblématiques, de par leur ampleur même, mais aussi en raison des effets de la publicisation de ces crises sur la réputation et la stratégie des firmes. Le désastre environnemental de la mine d'or et de cuivre d'Ok Tedi en Papouasie Nouvelle-Guinée, exploitée entre autres par BHP, et dont les ravages ont été identifiés dès les années 1980, est devenu une affaire publique dans les années 1990 (voir Ballard and Banks, 1997 ; Hyndman, 2005; Kirsch, 2006). Les fuites en mars 1996 dans la digue de résidus contenant des produits toxiques dans les rivières en contrebas de la mine d'or de Marcopper détenue par la firme canadienne Placer Dome aux Philippines ont également marqué les esprits (Dashwood, 2013 : 137, 141 142 ; Chaloping-March ici ${ }^{2}$. La firme Placer Dome a d'ailleurs été montrée du doigt pour des dégâts causés par les produits chimiques utilisés pour l'extraction de l'or du minerai dans les mines de Misima et Porgera en Papouasie Nouvelle-Guinée. Par ailleurs, la violence sociale générée autour de la mine de Porgera, dont John Burton nous rappelle ici (pp. 37-51) l'acuité et la persistance (voir aussi Filer, 1999), montre bien les limites des dispositifs mis en place par les firmes (dans le cadre d'un discours de responsabilité sociale d'entreprise; voir Filer et al., 2008 ; Dawshwood, 2013) et les États (les Development Forums instaurés en Papouasie Nouvelle-Guinée à partir de 1989 et analysés par Filer, 2012). La mine d'or et de cuivre de Grasberg en Papouasie indonésienne constitue quant à elle un exemple paradigmatique de collusion clientéliste, en l'occurrence entre la firme texane FreeportMcMoRan et les plus hauts niveaux de l'État et de la hiérarchie militaire en Indonésie, sans que la transition démocratique post-Suharto en 1998 ait malheureusement apporté de grand changement à cet égard (Ballard and Banks, 2009). Ce "branchement" direct entre firme et arène étatique nationale - forme d'enclave institutionnelle et politique - contribue de manière brutale à la reproduction de l'enclave minière physique, via la mise au service de son fonctionnement des forces de répression locales : violences environnementales, sociales et politiques se renforcent ici mutuellement à un niveau rarement égalé (Leith, 2003). Enfin, le cas de la mine de cuivre de Panguna sur l'île de Bougainville, toujours en Papouasie NouvelleGuinée, symbolise tragiquement le télescopage d'une crise environnementale, sociale et politique débouchant sur une guerre civile meurtrière (voir en particulier Filer, 1990).

2. Les mots employés pour qualifier ces événements font aussi partie du processus de mise en récit et des controverses qui l'accompagnent. Il a fallu attendre 1999 pour que BHP décrive les dommages causés par l'exploitation d'Ok Tedi comme un «désastre environnemental » (Asia Times online du 13 août 1999; http://www.atimes.com/oceania/AH13Ah01.html). Dans le cas de la rupture de la digue contenant les résidus chimiques de l'exploitation aurifère à Omai au Guyana, la communauté environnementale parlait de "catastrophe environnementale " tandis que les ingénieurs, consultants et firmes minières en faisaient un « incident environnemental » (Dashwood, 2013 : 141). 


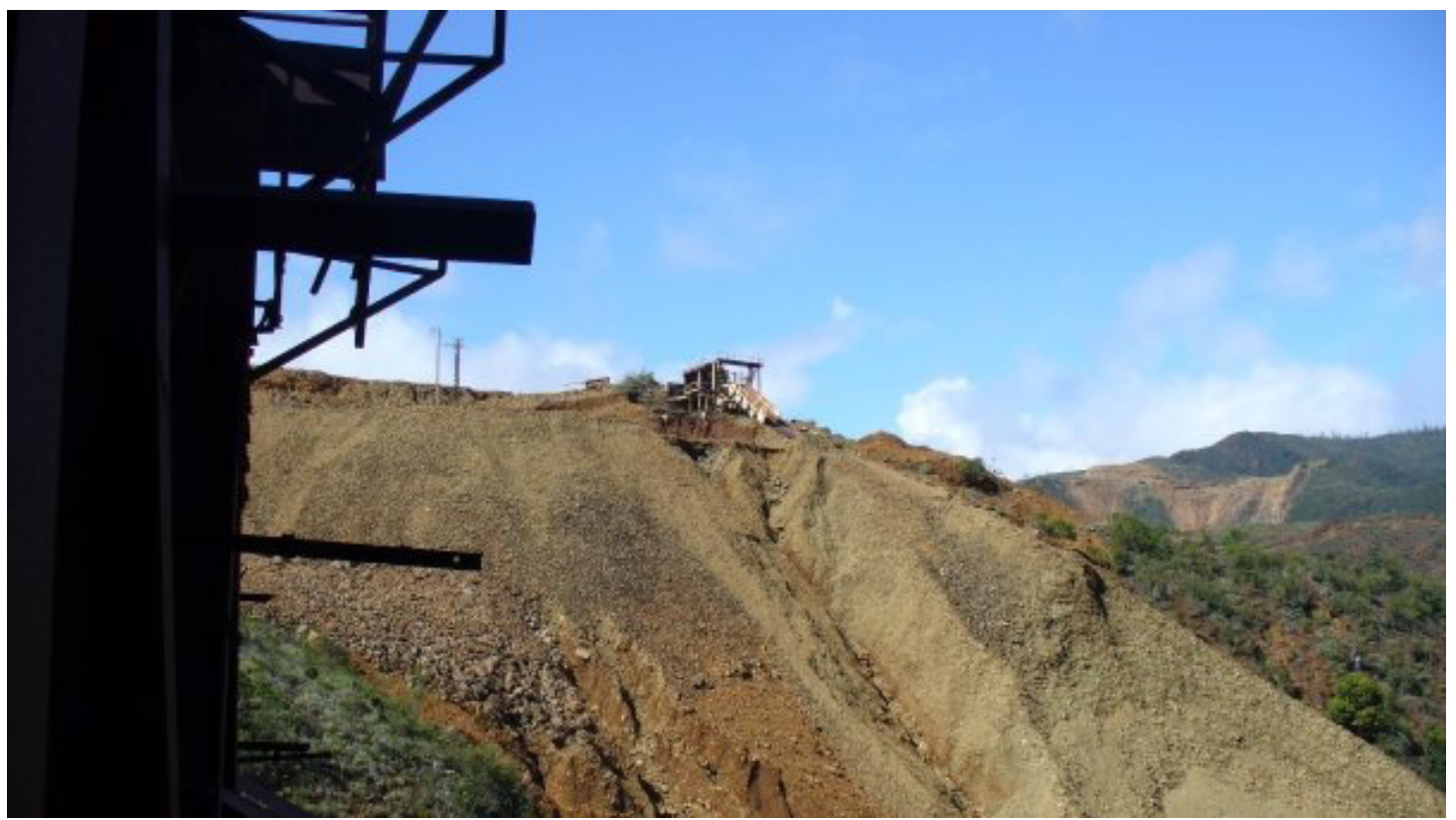

Рното 1. - Mine abandonnée Kaa Wi Paa de Kouaoua (Nouvelle-Calédonie) (2008, cliché Eddy Banaré)

En Nouvelle-Calédonie, l'impact environnemental de l'exploitation minière a fait l'objet de fortes controverses, y compris au sein du monde scientifique (Richer de Forges et Pascal, 2008) ${ }^{3}$ tandis que sa contribution au développement et à la décolonisation du pays était également soulignée (Grochain et al., à paraître). Le cas de Nauru examiné par Nancy Pollock dans ce dossier (pp. 107-119) apparaît enfin comme la combinaison dramatique d'un désastre environnemental et d'un échec du développement. L'activité minière a aussi généré des problèmes fonciers et politico-culturels, lorsque l'implantation des mines affectait des territoires à forte teneur symbolique et patrimoniale. En d'autres termes, et à l'instar du foncier, la mine est "affaire de pouvoir, de richesse et de sens » (Shipton and Goheen, 1992). Elle marque durablement de son empreinte les sociétés océaniennes contemporaines, mettant en jeu l'avenir des populations sur un mode bien souvent conflictuel. La mine comme activité et comme projet se construit dans la confrontation, confrontation de l'homme avec la nature, mais aussi confrontation de points de vue, de représentations, de stratégies, de logiques d'action, d'interprétations des enjeux qu'elle porte ou transforme.

La pratique minière est inséparable de sa mise en récit: mise en récit d'un passé non minier, d'un présent magnifié ou difficile, d'un futur aux contours souvent très incertains, parfois trop bien dessinés. Les représentations du travail et des effets de la mine dans l'histoire et dans la littérature peuvent nous apporter des éclairages très révélateurs sur la manière dont la mine a modelé les imaginaires et les représentations des populations et des institutions concernées par cette activité. L'enjeu temporel, par exemple selon qu'on se situe dans l'avant, le présent ou l'après de la mine, ou encore en fonction de la profondeur historique de la présence minière dans un endroit, joue fortement sur l'interprétation, la compréhension et les attentes locales (voir Polier, 1996 ; Imbun, 2011 ; voir aussi Nash, 1993, pour son livre fondateur sur le cas bolivien, et Taussig, 2010).

Nous nous intéressons ici à l'ensemble des types de récits générés par cette activité, qu'ils soient issus des enquêtes de terrain, des documents de politiques publiques, des sources archivistiques ou de la création littéraire. En quoi l'activité minière participe-t-elle des grands récits communautaires et/ou nationaux dans le Pacifique? Comment contribue-telle au renouvellement ou à la réactivation de récits et mythes d'origine et à l'élaboration des mémoires collectives ? Dans quel contexte, et en fonction de quels enjeux passe-t-elle d'un statut d'activité économique aux effets négatifs sur l'environnement à celui de patrimoine historique, valorisé comme une extension mémorielle et matérielle d'un collectif? En bref, de quelle manière et à quelle échelle (locale, nationale,

3. Voir en particulier les controverses internes au centre IRD de Nouméa entre 2004 et 2008, qui ont abouti à l'éclatement d'une unité de recherche, controverses que Julien Merlin retrace et analyse dans sa thèse en cours, doctorat en anthropologie que l'un de nous (P.-Y. Le Meur) codirige avec Madeleine Akrich, directrice du Centre de sociologie de l'innovation de Mines-ParisTech. 
globale) la mine nourrit-elle, ou au contraire, empêche-t-elle la création de ce que Benedict Anderson (1983) nomme les "communautés imaginées " (voir aussi Foster, 1995, 2002 pour la Mélanésie postcoloniale)? On verra que les textes rassemblés ici répondent chacun de manière partielle à ces questions politiques, en fonction du niveau d'analyse privilégié. Ainsi, la question du "nationalisme des ressources" traitée par Minerva Chaloping-March (pp. 93106) se résout aux Philippines dans le cadre de controverses qui opposent l'État central aux échelons politico-administratifs provinciaux et locaux alliés à des groupes de la société civile, quant à la bonne définition du bien commun auquel le développement minier est supposé contribuer. Un tel débat, qui concerne les formes de "localisation " de l'activité minière et de ses effets, se retrouve en Papouasie NouvelleGuinée, mais il se développe dans des espaces narratifs modulables, allant du local au global, comme le montre Dan Jorgensen en particulier.

\section{Récits miniers : éléments constitutifs}

Parler de « récit » ou de « mise en récit » implique une clarification d'un terme potentiellement polysémique. Dans Le nouveau discours du récit, le narratologue Gérard Genette distingue trois sens. Le récit peut désigner soit "l'énoncé narratif, le discours oral ou écrit qui assume la relation d'un événement ou d'une série d'événements ", soit «la succession d'événements, réels ou fictifs, qui font l'objet de ce discours, et leurs diverses relations", soit "l'acte de narrer en lui-même" (2007: 13-14). Genette choisit de restreindre l'usage du terme "récit» au premier sens, "signifiant, énoncé, discours ou texte narratif ». Il nomme " histoire " ou encore " diégèse " (ibid. : n15, 301-302) le second, à savoir un « signifié ou contenu narratif ", et "narration " le troisième, qui correspond à « l'acte narratif producteur et, par extension, l'ensemble de la situation réelle ou fictive dans laquelle il prend place" (ibid. : 15).

L'objet d'étude de Gérard Genette est la littérature (en l'occurrence, dans ce livre, $\grave{A}$ la recherche du temps perdu de Marcel Proust) et, plus précisément, le récit au sens restreint :

« [D] es trois niveaux distingués à l'instant, celui de discours narratifs est le seul qui s'offre directement à l'analyse textuelle, qui est elle-même le seul instrument dont nous disposions dans le champ du récit littéraire, et spécialement du récit de fiction. » (ibid.)
Le champ d'investigation ouvert par le présent dossier est plus large et il est clair que les auteurs rassemblés ici mobilisent d'autres outils que le texte - et donc le récit comme signifiant (le premier sens retenu par Genette) - pour étayer leurs discours : traces archéologiques, matériaux ethnographiques, sources archivistiques, données scientifiques, installations artistiques.

Dans la seconde partie du même ouvrage (Le nouveau discours du récit), écrite une décennie après la première partie (Figures III : Discours $d u$ récit), Gérard Genette revient sur les critiques adressées à ce premier texte. Il précise en particulier l'articulation entre récit, histoire et narration, selon que l'on se situe dans le registre fictionnel ou non.

"L'ordre véritable, dans un récit non fictif (historique, par exemple), est évidemment histoire (les événements révolus) - narration (l'acte narratif de l'historien) - récit : le produit de cet acte, éventuellement ou virtuellement susceptible de lui survivre en texte écrit, en enregistrement, en mémoire humaine. » (Genette, 2007 : 298)

\section{Il ajoute :}

"Cette rémanence seule, en fait, autorise à considérer le récit comme ultérieur à la narration : dans sa première occurrence, orale ou même écrite, il lui est parfaitement simultané, et leur distinction est moins de temps que d'aspect, récit désignant le discours prononcé (aspect syntaxique et sémantique, selon les termes de Morris), narration la situation dans laquelle il est proféré : aspect pragmatique. » (ibid.)

On pourrait avancer que c'est précisément dans cet espace que se déploie ce que l'on appelle classiquement en historiographie la « critique des sources " : la compréhension (partielle) du récit par l'analyse de ses conditions de production, qui permet, concernant une source directe tout comme un texte sociologique, de tester la "plausibilité " d'une interprétation (Passeron, 1991). Revenons à Gérard Genette :

« En fiction, cette situation narrative réelle est feinte [...], mais l'ordre véritable serait plutôt quelque chose comme narration $\rightarrow$ histoire/récit, l'acte narratif instaurant (inventant) à la fois l'histoire et son récit, alors parfaitement indissociables. "(Genette, 2007 : 298-299)

La construction des séquences narratives portant sur des situations fictionnelles ou historiques ${ }^{4}$ diffère donc, même s'il s'agit bien sûr d'idéaltypes : " [e]xista-t-il jamais une pure fiction? Et une pure non-fiction?" (Genette, 2007 : 299; voir aussi Ricœur, 1983-1985). étudient. 


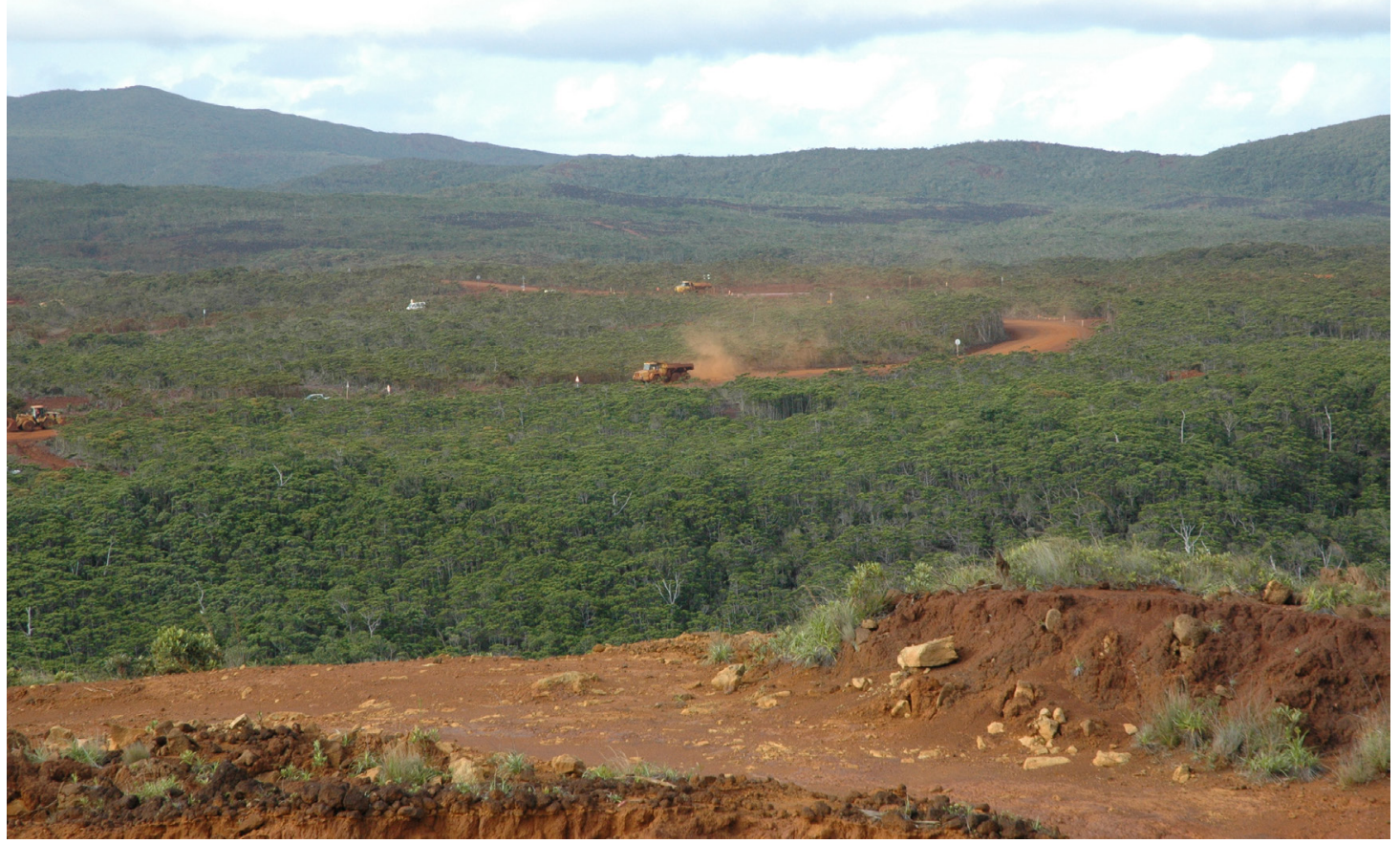

Рното 2. - Roulage sur paysage minier à Goro (sud-est de la Grande Terre, Nouvelle-Calédonie) (avril 2007, cliché Isabelle Leblic)

Cette frontière floue nous intéresse ici, interface où se développent des notions telles que celle d'identité narrative sur laquelle nous allons revenir, ou encore la question de l'interprétation dans le domaine des sciences sociales. Nous rejoignons ici Jean-Pierre Olivier de Sardan lorsqu'il affirme que :

"[l']espace épistémologique propre aux sciences sociales est [...] à la fois intégralement interprétatif et empiriquement contraint." (Olivier de Sardan, 2008 : 266-267)

Dans ce cadre, la légitimation de tout énoncé interprétatif se fonde sur un certain "indice de véridicité ", un lien au "réel " que l'on peut retracer (ou contester). Cela admis, l'ouverture du présent dossier minier aux études littéraires et représentations artistiques permet d'élargir le propos et de mobiliser le corpus théorique proposé par les spécialistes du récit littéraire et de la sémiotique. Eddy Banaré suggère dans son article sur la littérature minière néo-calédonienne (pp. 151-164) que les études littéraires peuvent se permettre, dans une logique intertextuelle, de confronter les points de vue :
" en prenant le parti, par exemple, de considérer que la vision des paysages miniers d'une poétesse kanak [...] pendant "les Evénements" (situation insurrectionnelle des années 1984-1988) pourrait être une réponse donnée à un chroniqueur colonial de la fin du XIX ${ }^{e}$ (p. 152)

Cette logique intertextuelle est par ailleurs très présente dans les récits présentés ici par Dan Jorgensen, en particulier dans la réécriture chrétienne du mythe d'origine d'Afek et l'analogie faite par certains informateurs entre la langue faiwol et celle de l'Ancien Testament ; on peut aussi identifier des indices d'intertextualité en filigrane dans l'article de James Leach. Il ne s'agit donc pas de s'abstraire de la chronologie historique comme Carlos Fuentes a pu se le permettre dans son extraordinaire Terra Nostra (1979), ni de plaider pour le plagiat par anticipation, à l'instar de Pierre Bayard (2009)5. L'enjeu est plutôt de repérer des récurrences ou des héritages, ou encore de situer des points de vue et d'identifier des dialogues potentiels au-delà de l'irréversibilité temporelle. Il faut également prendre en compte l'«écologie historique " (Clifford, 2013: 42) dans laquelle évoluent les peuples autochtones (et sans doute

5. Avec la citation et l'allusion, le plagiat est l'une des trois formes d'intertextualité reconnues par Genette dans son acception restreinte de l'intertextualité comme relation de coprésence de deux ou plusieurs textes, de présence d'un texte dans un autre (Montalbetti, 1998 : 64). 
pas seulement eux), une longue durée marquée par une double orientation vers le passé et l'avenir, à la base de l'image de la spirale forgée par Epili Hau'ofa (1993). Ian McIntosh montre ainsi dans une étude consacrée aux Yolngu de la terre d'Arnhem en Australie du Nord que l'ancrage à la fois historique et mythique de leurs rapports sur la longue durée à l'enjeu minier, en particulier dans le cadre de relations débutées au tournant des XVIII ${ }^{\mathrm{e}}$ et $\mathrm{XIX}^{\mathrm{e}}$ siècles avec des pêcheurs provenant de Sulawesi, a fortement contraint l'interprétation de l'irruption contemporaine de l'industrie minière :

"There is a complete subordination of history to the ideology of Dreaming. [...] To follow the law and realise a pre-ordained future, mining must occur, but only on aboriginal terms." (McIntoch, 2004: 26 ; voir aussi Jorgensen, 1990 et infra)

Pour aller plus loin dans la mise en dialogue des sciences sociales avec le champ artistique et littéraire, nous continuerons de mobiliser les catégories proposées par Genette dans son discours sur le récit empruntant à la grammaire du verbe (Montalbetti, 1998 : 50-56) : temps, mode, voix. Le temps comprend l'ordre - qui s'intéresse aux éventuels décalages ou anachronies entre la succession des événements dans l'histoire et leur représentation dans le récit -, la durée ou vitesse - qui exprime les différences de rythme entre histoire et récit-, et la fréquence- les récits pouvant être plus ou moins répétitifs ou itératifs. Le mode renvoie à la distance entre le narrateur et l'événement - distance maximale dans un discours narrativisé, minimale dans un discours rapporté - et la perspective (ou focalisation) adoptée. En d'autres termes, le narrateur en dit plus ou moins que ce que sait le personnage, ce qui pose, entre autres questions, celle, classique en littérature, du narrateur omniscient ( focalisation zéro »). La question du mode peut aussi concerner les sciences sociales, du moins certaines approches d'inspiration structuraliste visant par exemple à révéler des structures ou des raisons inconscientes aux acteurs sociaux. La voix renvoie à l'identification de l'instance narrative qui n'est pas nécessairement l'auteur
(Genette, 2007 : 219-222). Elle est pour partie affaire de temporalité puisqu'elle situe dans le temps le récit par rapport à son référentiel : ultérieur le plus souvent (on raconte une histoire passée), antérieur dans le cas d'un récit prédictif, simultané, ou encore intercalé, lorsque le récit se développe entre des moments de l'action. Genette distingue aussi dans cette catégorie de la voix des niveaux de narration, avec la possibilité d'imbrication d'un récit dans un autre. L'opposition principale se situe entre le niveau extradiégétique du narrateur extérieur au récit et le niveau diégétique (ou intradiégétique) des récits des personnages ${ }^{6}$. La seconde opposition, qui nous intéresse particulièrement ici, survient entre récit homodiégétique, lorsque le personnage-narrateur prend lui-même part aux éléments du récit qu'il raconte (s'il en est le héros, on parle de récit autodiégétique), et récit hétérodiégétique lorsqu'il ne participe pas à l'histoire dont il fait le récit?.

Les flottements des récits anthropologiques, entre relation des actes et paroles des personnes enquêtées et intrusion plus ou moins discrète de la figure de l'anthropologue, correspondent à la paire hétéro/ homodiégétique, voire autodiégétique, pour ceux qui oublient l'aphorisme de Smith cité par Sanjek : "The subjects of ethnographies, it should never be for-
gotten, are always more interesting than their authors."
$(1990: 610)$

Lautre tension qui structure les récits ethnographiques a été décryptée avec acuité par Johannes Fabian; elle concerne le thème de l'allochronie, et plus spécifiquement ce qu'il nomme le "déni de co-temporalité " des récits anthropologiques (2006: 79) générateur d'une relation schizogène au temps (ibid.: 77) : la co-temporalité constitutive du terrain ethnographique est gommée dans des mises en récit qui sont tout autant des mises à distance de "l'autre », voire un refus de son historicité. Si l'écriture ethnographique a su évoluer depuis ce livre (Clifford and Marcus, 1986 ; Dawson et al., 1997 ; voir aussi Fabian, 2006) ${ }^{8}$, avec en particulier la naissance d'une anthropologie dialogique, attentive aux situations d'interlocution" (voir

6. Le niveau métadiégétique correspond à la prise en charge d'un récit à l'intérieur de l'histoire ou diégèse, pouvant donner lieu à une série d'enchâssements narratifs (on pense ici aux Mille et une nuits ou à Si par une nuit d'hiver... d'Italo Calvino). Genette distingue finalement six types fonctionnels dans les relations entre le niveau métadiégétique et le récit premier (extradiégétique) dans lequel il s'insère : fonction explicative, prédictive, thématique, persuasive, distractive, obstructive (Genette, 2007 : 365-366 ; 241-243 pour une première typologie en trois classes).

7. Voir aussi Davies (1999: 218-219) qui parle de métarécit pour qualifier le travail d'organisation de récits primaires pluriels que réalise l'écriture ethnographique.

8. C'est également vrai de l'écriture historique qui a été traversée par de profonds débats en la matière, en particulier quant à la relation entre la notion de récit et le refus - puis le retour - de l'événement (voir en particulier Burke, 1991 ; Hartog, 2003 : 202-215 et 2005 ; White, 2009).

9. La thématique de la rhétorique, du discours comme constitutif de la société a été approfondie dans l'anthropologie océaniste (Brenneis and Myers, 1984; Gegeo and Watson-Gegeo, 1990) mais pas dans le champ minier et du point de vue plus narratif abordé ici. 
Masquelier et Siran, 2000), on verra que les récits forgés dans le cadre de discours de légitimation de politiques publiques, en l'occurrence de politiques minières, fonctionnent selon des mécanismes proches de ceux décrits par Fabian. Une différence essentielle tient à l'identification du narrateur et surtout de l'auteur. Il nous faut ici étendre notre réflexion en direction d'usages non plus littéraires, mais philosophiques, politiques et anthropologiques de la notion de récit.

\section{La mine entre grands récits et petites histoires}

La notion de "grand récit " a été mobilisée par ceux-là même qui en annonçaient la fin, à savoir des philosophes qualifiés par commodité de " postmodernes » comme Jean-François Lyotard :

« En simplifiant à l'extrême, on tient pour "postmoderne" l'incrédulité à l'égard des métarécits. » (Lyotard, $1979: 7$ )

$\mathrm{Ou}$, comme l'énonce de manière lapidaire Emery Roe dans son livre sur les policy narratives, et en référence à Lyotard : "Metanarratives have gotten a very bad press lately" (1994: 52 ; voir aussi Clifford, 2013 : 40-41). Lyotard s'intéresse surtout au rôle de la fonction narrative dans la légitimation du savoir scientifique, dont les ressorts peuvent être d'ordre politique ou philosophique (1979: 49 sq.). Les grands récits des lumières, de la modernisation ou du développement peuvent être interprétés en termes de relation au savoir (le savoir scientifique de l'époque de la genèse du récit, souvent peu actualisé, se sédimentant peu à peu en substrat idéologique), mais aussi de rapport au temps: " $A$ modern future set against a primitive present", comme l'énonce Frederick Cooper (1997: 65) à propos du développement colonial (et postcolonial) comme pratique discursive.

La justification de l'exploitation minière s'est souvent traduite par de tels discours du développement et de la modernisation, que ce soit à l'époque coloniale ou après. Yann Bencivengo (pp. 137-149) montre bien comment la Société Le Nickel a émergé en Nouvelle-Calédonie à la jonction de plusieurs trajectoires : celle de la naissance de l'industrie du nickel, celle de l'histoire coloniale française et celle, spécifique, de la Nouvelle-Calédonie, comme lieu de colonisation de peuplement, de ségrégation raciale et spatiale et d'importation de main d'œuvre extérieure ${ }^{10}$. Les récits miniers, que ce soit ceux des prospecteurs individuels ou d'une future entreprise hégémonique sur le territoire néo-calédonien, comme la SLN, ou encore ceux, littéraires, évoqués par Banaré, se situent à la croisée de ces trajectoires (voir aussi Banaré, 2012, 2014).

À ce stade, la distinction entre récit (narrative) et histoire (story: c'est la diégèse de Genette, cf. supra) proposée par Sivaramakrishnan nous est utile :

"[...] development - as it is imagined, practiced, and re-created - is best described as stories that can change in their telling, as they are pieced together into contingently coherent narratives. Development's stories are rife with a micropolitics often obscured by the consistency or more orderly progression implied by the terms discourse or narrative. " (Sivaramakrishnan, $2000:$ 432)

Les récits des pionniers qu'ils soient directs, rapportés (par la presse de l'époque) ou encore mis en forme (et en fiction) par des écrivains sont autant d'histoires, hétérogènes, individuelles, comme celle de Higginson, l'un des fondateurs de la SLN, racontée par Thompson (2000). Ils présentent toutefois certains traits récurrents qui relèvent du "récit colonial ", celui de la réussite du défricheur (blanc, homme) «mettant en valeur" des terres neuves ("vacantes et sans maître ") et de la mission civilisatrice occidentale. L'identification de l'auteur des histoires spécifiques paraît simple, oscillant entre les postures hétéro et homodiégétiques, tandis que le récit colonial en semble dépourvu ${ }^{11}$. Ça ne veut pas dire que ces récits sans auteur sont dépourvus de narrateurs (et de narrataires, au sens de Genette : cibles visées par les narrateurs), et c'est tout l'enjeu de l'indexation sociale et politique de ces récits, de leurs usages et des logiques stratégiques et représentationnelles dans lesquelles ils s'inscrivent.

Les récits de politique publique (policy narratives) font partie des récits sans auteur relevant d'une analyse en termes de structuration (ou de morphologie) et, pour reprendre le terme de Propp, 1970), de fonctions ${ }^{12}$. Les récits de politique publique se présentent sous la forme d'un enchaînement narratif de relations causales visant à soutenir une certaine thèse ; leur fonction est de légitimer une orientation politique (Roe,

10. Voir aussi Bencivengo (2012) pour le cas particulier de l'importation de main-d'œuvre japonaise en Nouvelle-Calédonie.

11. Un peu à la manière dont le droit se construit comme régime d'énonciation qui a "comme réalité propre de précéder tout énonciateur " (Latour, 2002 : 298).

12. Propp place au centre de son analyse de la morphologie du conte les "fonctions " structurales qui organisent les contes: "Par fonction, nous entendons l'action d'un personnage, définie du point de vue de sa signification dans l'intrigue" (Propp, $1970: 31$ ). Il ajoute : " [L]es contes attribuent facilement les mêmes actions aux hommes, aux choses et aux animaux " (1970: 12); on est ici proche de l'actant du sémioticien Greimas (voir Greimas et Courtès, 1993 : 3-4), repris par la sociologie de l'acteur réseau de Latour et Callon. 
1994 ; Mosse, 2004). Emery Roe précise leur rôle de stabilisation d'un argumentaire à des fins de (justification de) décision dans des contextes marqués par l'incertitude, la complexité ou la polarisation de la question traitée (1994: 3). Il part d'une définition simple du récit comme histoire ou diégèse (story) :

"If they are stories, they have beginnings, middles and ends, as in scenarios; if the stories are in the form of arguments, they have premises and conclusions. " (Roe, 1994 : 3)

"Each relates a succession of events, real or hypothetical.» (ibid. : 53)

Roe complexifie ensuite la définition du récit en ajoutant à la catégorie "histoire" celle de " non-histoire» (nonstory), "contre-histoire " (counterstory) et "métarécit" (metanarrative), avec un petit " $\mathrm{m}$ " comme il le précise à plusieurs reprises (par opposition aux Métarécits ou "grands récits » de la raison ou de la science, par exemple; ibid: 12). Une contre-histoire constitue un récit alternatif ou opposé au récit dominant, tandis qu'une non-histoire diffère par sa structure, qui n'est pas celle, linéaire, du récit, et recouvre par exemple les arguments circulaires. Le métarécit est un récit de second ordre déduit par l'analyste à partir de la comparaison des différentes histoires, contre-histoires et non-histoires constitutives d'une controverse politique. Roe voit dans ce métarécit un nouveau récit potentiel qui permettrait de dépasser les contradictions des histoires et contre-histoires et de construire un argumentaire plus ajusté à la question politique en jeu :

"Today's metanarratives are tomorrow policy narratives, to be superseded at a later date. "(ibid. : 17)

Prenons un exemple traité par Nancy Pollock (pp. 107-119) dans le présent dossier, à travers l'exploitation des phosphates à Nauru : la thèse de la malédiction des ressources (resource curse). Il s'agit de l'un des plus puissants récits de politiques publiques en vigueur, tout particulièrement concernant l'enjeu minier. Pollock propose un récit du désastre environnemental et social généré par l'exploitation du phosphate à Nauru constitué d'un enchaînement causal que la notion de malédiction des ressources permet de mettre en forme sur un mode un peu téléologique, dans la mesure où la fin (provisoire) de l'histoire est déjà connue. Ce récit s'inscrit dans une généalogie coloniale mobilisant à la fois le discours de la terra nullius (c'est-à-dire sans maître suffisamment civilisé pour être reconnu comme tel), au besoin en construisant ce territoire vacant par le biais de déplacements de populations (mécanisme également exploré par Julia Edwards à Banaba (pp. 121-136 ; voir aussi Shlomowitz and Munro, 1992) ${ }^{13}$ et une politique d'importation de main-d'œuvre, mise en œuvre en Nouvelle-Calédonie également (comme le montre Bencivengo, ici et 2012 ; Le Meur, 2012). La mise en forme discursive du récit minier comme expression d'une malédiction des ressources a été largement discutée depuis la fin des années 1980 (Rosser, 2006 : 7 ; Robinson et al., 2006) comme tentative de capturer les effets multiples et en particulier les impacts négatifs de la mine sur les économies locales et nationales, en termes de performance économique, de mauvaise gestion, de conflictualité et de type de régime politique. La thèse de la malédiction des ressources a connu un large succès dans le monde des praticiens et des économistes du développement international, car elle propose un modèle repoussoir simple permettant la promotion des solutions développementistes. Ce modèle tend à télescoper des processus hétérogènes dans le lit de Procuste d'un cadre normatif et explicatif unique qui masque les spécificités des trajectoires historiques locales et nationales. Derrière la catégorie fourre-tout (et peu sociologique), il faut décrypter des rapports de force entre acteurs, des collusions et des alliances, des logiques rentières des États, des manières, pour les multinationales minières, de se construire comme enclave physique mais aussi institutionnelle, des réponses et réinterprétations locales, etc. (Le Meur et al., 2013). Ces différents ingrédients constituent l'économie politique de la mine, et la malédiction des ressources n'en est pas seulement le voile, mais aussi un actant - "a force in themselves" (Roe, 1994:2) - au sens où le mot légitime des choix politiques, en particulier en laissant hors du champ le rôle des entreprises minières et de leurs pays d'origine, comme le montre bien le cas de Nauru. L’ouverture de la « boîte noire » que constitue le discours sur la malédiction des ressources ouvre ainsi la voie (ou la voix) à des récits alternatifs, des contre-histoires au sens de Roe, inversant en valeur la malédiction des ressources pour en faire une opportunité, sous réserve de prendre en compte les éléments clefs du contexte sociétal (Bebbington et al., 2008 ; O'Faircheallaigh, 2012).

Malédiction des ressources ou opportunité, les deux faces de ce récit croisent dans le champ politique d'autres récits, comme celui analysé par Minerva Chaloping-March (pp. 93-106) pour les Philippines. La notion de "nationalisme des ressources" (resource nationalism) apparaît

13. On pense aussi à l'enfermement des Kanak dans des réserves par la colonisation française, voir entre autres Saussol (1979), Leblic (1993), Merle (1998). 
comme un récit de construction nationale, éventuellement de construction d'un État rentier, dans un contexte capitaliste globalisé, dont la force varie entre autres avec les cycles des prix des matières premières (voir Bremmer and Johnston, 2009; Ward, 2009). ChalopingMarch déplace la problématique en direction de la relation entre national et local, ou plutôt entre souveraineté et citoyenneté. La question qu'elle pose peut être formulée ainsi : dans quelle mesure le récit d'une indépendance nationale basée sur une exploitation contrôlée des ressources minières fait-il l'objet d'un débat politique national et est-il partagé par une base sociale large ? On retrouve ce type de questionnement en Nouvelle-Calédonie où, depuis la fin des années 1990, les indépendantistes ont placé au centre de leur stratégie politique le contrôle économique sur l'extraction et la transformation du nickel ( $c f$. Grochain, 2013 ; Grochain et al., à paraître), et où les leaders politiques " loyalistes " s'emparent de manière a priori surprenante de l'argument de la prise de participation dans l'industrie minière.

Dans le cas philippin, le nationalisme des ressources fonctionne comme une sorte de métarécit structuré par des controverses entre récits et contre-récits, qui constituent aussi des points d'articulation entre groupes d'acteurs et échelons politico-administratifs nationaux et intra-nationaux. À l'enchaînement causal pensé à plan national [libéralisation économique $\Rightarrow$ investissements miniers $=>$ croissance $=>$ développement durable $=>$ justice et équité] répond un discours de contrôle local sur l'exploitation des ressources et la préservation de l'environnement émanant de coalitions et réseaux politiques qui invoquent eux aussi l'argument du nationalisme des ressources. ChalopingMarch montre aussi très clairement le rôle d'événements pivots (désastre environnemental ou nouvelles régulation minière) dans la construction des récits de politique publique. Dans la même veine nationaliste, mais sur un registre nettement patrimonial cette fois, les découvertes archéologiques mettant en évidence l'ancienneté et la continuité d'une activité minière d'origine précoloniale permettent d'ancrer une exploitation minière récente et opérée par une firme chinoise dans un récit national légitime, comme dans le cas laotien étudié par Mayes et Chang (2013). Nakoro (2011), dans un texte sur les relations complexes entre fouilles archéologiques et exploitation minière à Fidji, décrit en revanche une situation d'opposition entre logiques de préservation patrimoniale et d'extraction minérale, tandis que Bainton et al., (2011) montrent comment le programme de gestion du patrimoine culturel issu d'un accord signé avec la firme exploitant la mine d'or de Lihir en Papouasie-NouvelleGuinée est manipulé dans les narrations et arènes politiques locales.

Évidemment, les industriels racontent aussi des histoires ${ }^{14}$. Comme le montre John Burton (pp. 37-51) qui revient dans sa contribution sur les controverses ayant accompagné l'exploitation de la mine de Porgera en Papouasie NouvelleGuinée (voir aussi Filer, 1999), les opérateurs miniers, sous la pression des gouvernements et/ ou de la société civile locale ou internationale, en sont venus à produire un impossible métarécit (au sens défini plus haut de synthèse de policy narratives différents, voire concurrents) qui tente de combiner maximisation du profit de la firme et développement durable des communautés, en particulier sous la forme du discours de la responsabilité sociale et environnementale des entreprises. Or l'examen de la pratique des firmes montre la fragmentation, voire la dilution des responsabilités derrière l'apparente cohérence du discours ( $c f$. Filer, Burton and Banks, 2008). Emery Roe note toutefois que les récits de politique publique - et le discours de type RSE ainsi que les régulations globales promues par les multinationales minières rassemblées au sein de l'Iсмм (International Council on Mining and Metals) sont des fragments de politique produits par des opérateurs privés - ne sont pas validés ou invalidés par la "réalité »:

"[ They] often resist change or modification even in the presence of contradicting empirical data, because they continue to underwrite and stabilize the assumptions for decision making in the face of high uncertainty, complexity, and polarization. " (Roe, 1994 : 2)

Les récits de politique publique, comme les "grands récits" (du développement, de la modernisation, etc.), perdurent en fonction du crédit qu'ils acquièrent et ce crédit est moins indexé à l'adéquation entre des faits et des interprétations qu'à la solidité des alliances constituées et à la force légitimatrice de la narration produite. C'est une affaire de degré, mais cet équilibre spécifique donne une couleur particulière aux controverses politiques par rapport aux controverses scientifiques ${ }^{15}$.

14. On pourrait ajouter ici les formes narratives émanant d'autres groupes d'acteurs dont le rapport au temps diffère : les géologues, inscrits dans un très long terme tellurique, les ingénieurs, contraints par l'urgence structurelle de trouver la solution au problème du moment. Nous remercions Michel Jébrak pour avoir attiré notre attention sur ce point.

15. Voir Callon (1981) sur les controverses technologiques, Latour (1989) sur les controverses scientifiques, Fabiani (1997) sur les controverses philosophiques. Albert Hirschmann, dans son essai sur la rhétorique réactionnaire (1991), montre bien comment les argumentaires peuvent s'inverser en valeur sans perdre de leur efficace. 


\section{Récits miniers, rhétorique et identité}

La section précédente a permis d'aborder, de manière parfois indirecte, le thème de la fonction des récits miniers. Il nous faut à présent poser la question de leurs fins. Dan Jorgensen (pp. 2336) propose dans sa contribution sur les récits et géographies multiples dans lesquels s'inscrit la trajectoire minière d'Ok Tedi en Papouasie Nouvelle-Guinée (voir aussi Hyndman, 2005), de distinguer les fonctions instrumentales ou stratégiques des récits (et mythes) et leurs fonctions non instrumentales, en s'appuyant sur le cas particulier d'un mythe d'origine aux développements inattendus. Les premières renvoient à la mobilisation et à l'ajustement de récits à des fins de justification d'un accès aux bénéfices de la mine. Les récits d'origine servent ainsi à fonder des revendications foncières portant sur les espaces d'une emprise minière actuelle ou à venir. Ces revendications peuvent prendre des formes non directement territoriales et renvoyer à des réseaux de clans ou de chefferie ou à des liens rituels (Jorgensen ici ; Horowitz, 2003 ; Brutti, 2007 ; Crook, 2007 ; Le Meur, Horowitz, Mennesson, 2013). La fonction justificatrice de ces récits réside aussi dans l'explication de la création et de l'existence de la richesse minérale (Biersack, 1999) renvoyant à une conception de la terre comme bien matériel et immatériel (Strathern, 2009).

La distinction proposée par Jorgensen entre fonctions instrumentales et non instrumentales des récits ouvre sur les deux questions des fins - pourquoi raconter? Pour persuader, négocier, décrire, (se) remémorer, exister ? - et du destinataire (ou narrataire) : à qui s'adresset-on lorsque l'on cherche à persuader, négocier, décrire, rappeler, exister?

Plusieurs articles dans ce volume mettent en avant, sur un mode instrumental, la volonté de contrôle du territoire et des ressources exprimée par des revendications et des politiques qui peuvent se situer au plan local (Jorgensen, Bacalzo et al.) ou national (Chaloping-March, Nanau). On peut traduire ces stratégies en termes de souveraineté, en déconnectant le concept de son usage traditionnellement associé à l'État sur la base d'une définition large et exploratoire: la souveraineté comme acte d' " autopositionnementé » :

" the ability of an entity to designate both its own identity and the scope of its authority. " (Joyce, 2013 : 35)

La souveraineté peut prendre des formes localisées, souvent, actuellement, dans le cadre de reformulations empruntant au discours de l'autochtonie, et elle alors parfois qualifiée de "souveraineté interne" dans des contextes de colonies de peuplement (Nouvelle-Calédonie, Australie, Canada, etc. ${ }^{16}$. À cet égard, le cas présenté par James Leach (pp. 53-62) est intéressant, car légèrement décalé par rapport à l'enjeu minier, et permettant de ce fait de répondre à la question posée par Jorgensen sur la dimension non instrumentale des récits associés à la mine. L'analyse de Leach mettant en avant la notion de "possession mutuelle " de la terre et du collectif humain (au-delà de la formule classique de l'homme appartenant à la terre) et le paysage comme processus animé et propriété émergente, déplace la problématique vers le domaine existentiel : les récits qu'il rapporte sont des récits de vie, de continuité de la communauté en tant que collectif, de persistance dans son être (allant au-delà, et étendant la portée de la notion de livelihood ou mode de subsistance). La sortie hors de la fonction instrumentale est en même temps sortie hors du registre de la propriété, devenu usuel dans les négociations entre populations locales et compagnies minières, comme le montre par exemple Colin Filer lorsqu'il évoque dans ce contexte, en Papouasie Nouvelle-Guinée, la montée d'une "idéologie foncière propriétariste" (2006; voir aussi Jorgensen, 2004). Celle-ci, résultant de l'obligation de réponse à l'injonction au développement des opérateurs miniers, peut toutefois prendre des formes plus ou moins exclusives ou inclusives, et qui varient également en termes de formes sociales et de schéma de parenté et de filiation, comme le rappellent Bacalzo Schwoerer et al. (pp. 63-76) dans l'introduction de leur article (voir aussi Golub, 2007). Ils insistent aussi sur la relation entre type de mise en forme de l'idéologie foncière et moment dans le cycle minier : l'hypothèse serait celle d'une tendance à l'inclusion plus grande de la part des populations locales dans les phases préliminaires de pré-exploitation.

Le texte de Jorgensen (pp. 23-36) laisse délibérément ouverte la question de la qualification des fonctions non instrumentales de certains récits miniers, ceux qui en particulier intègrent de manière étonnante des éléments combinant le mythe des tribus perdues d'Israël et des résultats de la génétique moderne. On pourrait avancer que la principale fonction non instrumentale de ces récits est existentielle: ils aident, en lien avec la persistance des pratiques et des savoirs qui les accompagnent, la communauté à perdurer concrètement et symboliquement dans son être tout en honorant

16. Voir en particulier Evans et al. (2013). Pour le lien entre autochtonie, souveraineté et enjeux minier en NouvelleCalédonie, voir Demmer (2007), Levacher (à paraître) ; Leblic (2007) et Graff (2012) reviennent sur les liens entre autochtonie, souveraineté et décolonisation dans ce même territoire. 
sa dette vis-à-vis de la nature (Esposito, 2000 ; Strathern, 2009). On pourrait bien sûr objecter qu'un récit justifiant de la vie d'un collectif revêt une fonction instrumentale. La réponse peut alors ici tenir dans la prise en compte de temporalités différentes, même si l'irruption brutale du capital global crée un effet de compression du temps. Analysant les logiques de l'échange, Parry and Bloch (1989) distinguent deux grands types d'ordres transactionnels, selon qu'ils s'inscrivent dans une durée inférieure ou supérieure à celle de la vie humaine. De manière analogue, on aurait d'un côté des récits plus instrumentaux et stratégiques utilisés pour négocier l'irruption de la mine et l'urgence qu'elle génère et de l'autre, des discours inscrits dans une durée et une continuité étendue. Ces décalages de temporalité sont analysés par Leach (pp. 53-62) dans une perspective analogue. Ces formes narratives ayant trait au mode d'existence d'un collectif peuvent aussi exprimer, comme le montre ici Jorgensen, la volonté d'un groupe social d'étendre son horizon au-delà du local, lorsqu'il s'inscrit dans l'universalisme chrétien par exemple ${ }^{17}$.

D'un point de vue pragmatique, la manière dont les récits circulent et se transforment montre de fortes imbrications entre registres instrumentaux et non instrumentaux. Cette imbrication obéit à une logique de bricolage et de mobilisation d'éléments hétérogènes, logique intertextuelle génératrice d'innovation narrative et non de répétition ${ }^{18}$. Cette logique d'assemblage n'empêche pas qu'un registre narratif puisse dominer à un moment dans l'organisation de ces récits miniers. C'est ce que nous observons dans une localité du sud de la Nouvelle-Calédonie (Thio, Borendi) marquée par une expérience séculaire et souvent conflictuelle d'exploitation du nickel. Or le récit de cette expérience par les acteurs locaux, en particulier dans ses moments de tension, mobilise un registre largement religieux (chrétien, mais pas seulement) imbriqué dans, et interprétant la trame événementielle ${ }^{19}$. À travers ces récits se construit une forme d' « identité narrative " comme forme de mise en cohérence dans la durée de l'action et de l'agent (Ricœur, 1990 : 137-139 ; Somers, 1994) ${ }^{20}$ qui à la fois organise une continuité existentielle du collectif et permet dans sa plasticité même de faire face, dans un rapport de force très inégal, aux bouleversements induits par l'irruption du fait minier. C'est ce que montrent en particulier Doris Bacalzo et al. (pp. 63-76) dans leur description fine des transformations des formes sociales locales (le "clan" en particulier) en réponse à l'obligation pour les populations de se positionner dans la compétition pour l'accès à une part de la rente minière. Le passage à des discours de justification moins inclusifs à mesure que la pression de l'enjeu minier s'accroît et se concrétise, comme évoqué ici, pourrait aussi s'interpréter dans une logique de continuum allant d'une réponse existentielle générale à un durcissement instrumental plus compétitif (sur ce point, voir aussi Bainton, 2009 ; Le Meur, à paraître).

La notion d'identité narrative reste en l'occurrence complexe à manipuler dans la mesure où les récits - modes de mise en intrigue par intrication causale - peuvent aussi, de par leur fonction même, contribuer à l'expression et à la définition de réseaux - acteurs réseaux ou dispositifs - visant à construire et légitimer des formes d'intéressement, de mobilisation et d'alliance (Latour, 2006). La composition du collectif se déplace en dehors du groupe autochtone pour inclure différents porte-parole, médiateurs et alliés de tous ordres. Cette voie, dont Bacalzo Schwoerer et al. (pp. 63-76) lancent l'exploration dans leur texte (voir aussi Horowitz, 2012), est prometteuse et mériterait un description plus systématique, permettant aussi de croiser ce point de vue avec des situations où les récits semblent plus directement indexés à des groupes d'acteurs nettement identifiés, comme dans le texte proposé ici par John Burton (voir aussi Le Meur, 2014).

\section{Récit et mise en récit : la question du narrateur}

Dans cette dernière section, nous allons revenir sur un point central volontairement laissé à l'écart jusqu'ici, à savoir celui du narrateur. Un récit suppose un narrateur et implique un narrataire, nous l'avons dit en première section de cette introduction : qui raconte et à

17. À l'inverse, Francesca Merlan (1994) parle de « récits de survie " dans son analyse des récits aborigènes contemporains rapportant la brutalité des rapports coloniaux en Australie du Nord.

18. Nous remercions Dan Jorgensen pour avoir attiré notre attention sur ce point.

19. Enquête en cours dans le cadre du programme NERVAL (Négocier, évaluer, reconnaitre la valeur des lieux), financé par le CNRT « Nickel et son environnement ».

20. Ricœur voit l'identité narrative comme expression de «la dialectique entre l'ipséité et la mêmeté " (1990 : 167), l'ipséité renvoyant à une réflexivité qui interroge la continuité temporelle au fondement de l'idée de mêmeté. Dans un texte antérieur, Ricœur donne la définition suivante de l'identité narrative (1985: 442), qui met en évidence la porosité entre récits historiographiques et littéraires (voir également White, 2009, sur ce point) : "Le rejeton fragile issu de l'union de l'histoire et de la fiction, c'est l'assignation à un individu ou à une communauté d'une identité spécifique qu'on peut appeler leur identité narrative ». 
destination de qui ? Il y a une intentionnalité du récit minier qu'il s'agit de situer par rapport à ce binôme narrateur/narrataire, dans un contexte historique donné, mais en se souvenant qu'une part de ces récits est sans auteur. On raconte pour faire agir, pour légitimer une présence et/ou une action, mais aussi, nous l'avons vu, à des fins non instrumentales, existentielles, mobilisant et contribuant à reproduire les récits sans auteurs déjà évoqués. Qui produit les récits miniers et dans quel but? La distinction principale à cet égard s'opère entre le narrateur acteur et le narrateur observateur. S'agit-il d'un témoignage direct écrit à la première personne ou d'une description produite par un observateur plus ou moins extérieur à la situation décrite? Les textes rassemblés ici sont souvent complexes de ce point de vue puisqu'ils incluent des éléments de récits rapportés ou enregistrés (niveau intradiégétique), écrits ou dits à la première personne, et aussi des analyses émanant des auteurs, qui eux-mêmes sont, dans le cas de récits ethnographiques, en situation d'observateurs participants. La proposition de Stuart Kirsch d'une anthropologie inverse (reverse anthropology) est celle qui apparemment va le plus loin dans l'effacement de l'anthropologue comme narrateur dans le but de faire la place la plus large aux histoires et interprétations locales :

«Le concept d'anthropologie inverse fut utilisé en premier par Roy Wagner [...] pour décrire la manière dont les cultes du cargo mélanésiens traitaient des notions capitalistes de profit, travail salarié et production. Wagner soutenait que les cultes du cargo constituaient la contrepartie interprétative de l'étude de la culture, et par conséquent, une sorte d'anthropologie inversée. Je développe cette analogie en examinant la façon dont les Yonggom déploient des analyses autochtones dans le cadre de leurs luttes politiques avec la compagnie minière et l'État. Je montre aussi dans quelle mesure les éléments dérivés des analyses autochtones peuvent contribuer aux débats politiques et théoriques contemporains sur ces enjeux. " (Kirsch, $2006: 3$, traduit par nous)

On retrouve ici l'un des modes de narration "ethnographique" évoqués par John Burton dans sa typologie des formes de mise en intrigue de la mine, qui n'oublie pas que les populations locales sont des acteurs historiques, politiques, réflexifs. Ce point pourrait paraître trivial si l'on ne constatait pas à quel point les récits produits par les acteurs directement concernés par l'enjeu minier sont souvent recouverts d'un voile narratif (extradiégétique) qui oriente la lecture et empêche d'en saisir tous les ressorts, y compris dans leurs incohérences et contradictions éventuelles.

L'écriture ethnographique comme travail d'organisation - de sélection, de découpage et de montage -, de récits issus du travail de terrain (cf. Davis, 1999, citée plus haut), peut aussi combiner dans une trame complexe des discours aux auteurs et narrateurs divers, comme le montre aussi John Burton. Ce dernier propose un récit partiellement homodiégétique, puisqu'il revient sur une polémique qui l'implique directement. En même temps, il mobilise dans son analyse non seulement les discours du développement et de la responsabilité sociale d'entreprise, mais aussi un récit cinématographique, le film Avatar, pour qualifier les positionnements d'autres acteurs et en particulier de l'ong Mining Watch qui le met en cause. La construction de son argumentaire repose sur imbrication d'une pluralité de modes narratifs.

Sur un autre registre, Florence Giulani (pp. 165-174) nous propose une mise en intrigue qui se déploie à deux niveaux. Elle raconte les tribulations d'un projet inspiré du Land Art sur une mine orpheline, projet voulu par la province Sud de la Nouvelle-Calédonie et dont l'ampleur se réduira sous la pression de diverses vicissitudes techniques et politiques. Elle nous donne ensuite à voir une installation artistique à plusieurs voix, puisqu'elle implique en particulier des femmes de la tribu de Saint-Louis. Celles-ci sont invitées à "panser ", grâce au tressage, la montagne écorchée par la mine. Ce faisant, elles se font les dépositaires d'une histoire qui est à la fois matérialisée par leur production et véhiculée par leur discours sur la mine.

Le jeu des histoires racontées et des récits mobilisés à des fins souvent stratégiques est l'objet d'interprétations socialement et politiquement indexées. Le prospecteur colonial travaille à la fois pour la grandeur de sa patrie et pour la sienne propre, en cherchant inlassablement à découvrir de nouveaux filons et à étendre le territoire de la "mise en valeur" minière. Ces deux niveaux de lecture sont identifiables mais l'auteur du rapport de prospection raconte son histoire, inscrite dans un espace et un temps localisés : on est bien loin de la quête du " temps aboli» que Thierry Hentsch (2005) voit au fondement des grands récits occidentaux (voir aussi Fabian, 2006). Le rapport de prospection décrit une série de "gestes" qui, pour Yves Citton, situe ce type de texte "à la croisée de la vie et de l'art dans la mesure où il représente une sensibilité esthétique inscrite dans la texture quotidienne de nos pratiques»(2012:56 ${ }^{21}$.

21. Aux récits et rapports de prospection coloniaux font échos les trajectoires contemporaines d'orpailleurs artisanaux en Afrique de l'Ouest et ailleurs, qui expriment, loin de tout exotisme, la dureté des sociétés qui se constituent sur les frontières minières, mais aussi les normes et les valeurs qui s'y forgent, à la base d'une " économie morale de l'orpaillage " (Grätz, 2004). 


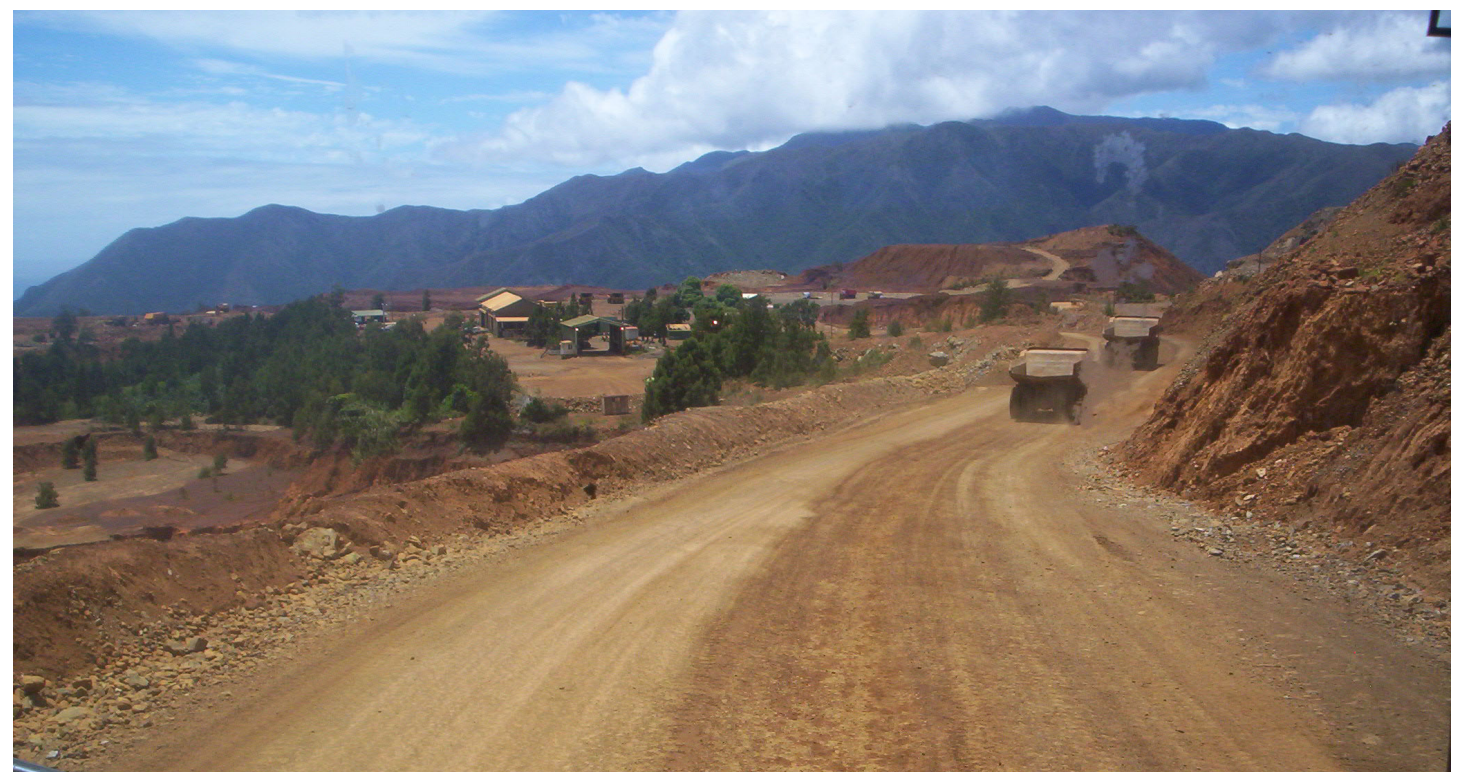

Рното 3. - Roulage sur mine à Thio (2008, cliché Marlène Dégremont)

L'écrivain kanak est quant à lui tiraillé entre les promesses de l'indépendance, le rêve d'un essor économique et la préservation d'une terre ancestrale, comme le montre Eddy Banaré ici. Quant à l'association écologiste, elle peut s'allier à une organisation autochtone pour la conservation de l'environnement tandis que cette dernière trouvera ensuite un terrain d'entente avec la firme minière autour de la notion du développement durable (Horowitz, 2012; Dashwood, 2013). La production de discours et d'histoires a donc souvent à voir avec l'exercice, la contestation ou la conquête d'un pouvoir qui passe par un travail sur l'histoire, au double sens de trame historique et narrative. Le récit, qu'il soit littéraire, historique ou à visée politique ou institutionnelle, assigne ou conteste des rôles; le processus est peut-être plus visible dans un contexte colonial de domination plus explicite.

«Le colon fait l'histoire et sait qu'il la fait. [...] L'histoire qu'il écrit n'est pas l'histoire du pays qu'il dépouille mais l'histoire de sa nation en ce qu'elle écume, viole et affame. » (Fanon, 2011 : 463)

Les rapports de prospection, les chroniques, les carnets de voyage ou encore les nouvelles, romans et poésies qui ont constitué les premiers corpus de récits miniers étaient écrits par des acteurs de la colonisation et destinés aux espaces coloniaux. En contexte postcolonial, on racontera, selon les perspectives, le courage des ancêtres pionniers, sortes de "héros culturels", ou le "trauma colonial " décrit par Patrick Sultan : le terreau mémoriel dans lequel viennent puiser les créateurs, et qui mêle "l'arrachement à un pays natal, la déportation de masse, la transplantation forcée, la destruction et la désorganisation des lieux ancestraux, la profanation des terres sacrées, [ou] la ségrégation" (Sultan, $2011:$ 107).

À un niveau supérieur d'agrégation, ces récits participent d'une écriture et d'une philosophie de l'histoire et, d'une certaine manière, de l'histoire elle-même. C'est cette voie qu'emprunte Hayden White lorsqu'il identifie une "imagination historique " (White, 1973, 2009) de l'Europe du $\mathrm{XIX}^{\mathrm{e}}$ siècle, qui se trouve être l'époque fondatrice des mythes pionniers des colonies de peuplement océaniennes. On peut la reconstituer à travers les chroniques, les rapports, la presse, tout un ensemble de récits dont l'écriture emprunte ses codes à différents genres littéraires, romance, tragédie, comédie ou satire. Il ne faut toutefois pas aller trop loin dans l'analogie :

"The notion that sequences of real events possess the formal attributes of the stories we tell about imaginary events could only have its origins in wishes, daydreams, reveries. " (White, $1990: 27$ )

\section{Conclusion}

L'activité minière est indissociable de déplacements de populations et de spoliations foncières, et elle génère une violence environnementale, sociale et politique parfois extrême. Or celle-ci est souvent masquée par le caractère épique des grands récits relayés par la presse coloniale ou dans des carnets de voyage, ou par le formalisme et l'apparence d'objectivité des rapports des firmes et des consultants consacrés au développement durable et à la responsabilité sociale d'entreprise. 
On peut avancer que l'une des fonctions des textes générés par l'exploitation minière est de circonvenir, au plan symbolique, mais aussi, de plus en plus, d'un point de vue institutionnel et juridique, les débordements environnementaux, économiques, politiques et sociaux souvent mal contrôlés de cette activité : conflits et guerres, accidents, faillites et effondrement des cours, corruption et clientélisme... Cette production de textes relève d'un double effort de rationalisation (l'exploitation minière comme entreprise calculée) et de poétisation (l'exploitation minière comme accomplissement individuel ou collectif). Ce corpus est aussi fait de non-dits (Barbançon, 1992), dont le plus assourdissant est celui généré par une parole coloniale qui " professe le silence de l'indigène" (Said, $2000: 161$ ) et travaille encore le présent des lieux d'exploitation minière et les récits qu'ils continuent de susciter. À travers ces récits, des empires "se sont rêvés", des mémoires et des représentations se sont forgées, constitutives des rapports aux sites et au travail miniers que les articles de ce dossier évoquent sur des registres variés.

Le récit comme discours sans auteur - " grand récit " ou métarécit, policy narrative - rencontre dans ces contextes divers le récit comme histoire racontée par un ou plusieurs auteurs situés, participant ou non à l'action qui est dite ou mise en scène. La mine dans le Pacifique constitue le substrat de trames narratives complexes et imbriquées, qui tentent, sur des registres différents allant de l'expérience individuelle à l'algorithme de politique publique, de dire les contradictions d'une activité qui transforme irrémédiablement les hommes et les paysages. Ce dossier rassemble des textes qui contribuent, chacun à leur manière et à partir de terrains variés (Fidji, Kiribati, Nauru, Nouvelle-Calédonie, Papouasie Nouvelle-Guinée, Philippines, Salomon), à l'expression, la mise en intrigue et en dialogue, de ces manières de raconter la mine dans le Pacifique.

\section{BIBLIOGRAPHIE}

Anderson Benedict, 1983. Imagined Communities. Reflections on the Origin and Spread of Nationalism, London, Verso.

Bainton Nicholas A., 2009. Keeping the Network Out of View: Mining, Distinctions and Exclusion in Melanesia, Oceania 79 (1), pp. 18-33.

Bainton Nicholas A, Chris Ballard, Kirsty Gillespie and Nicholas Hall, 2011. Stepping Stones Across the Lihir Islands: Developing Cultural Heritage Management in the Context of a Gold Mining-Operation, International Journal of Cultural Property 18, pp. 81-110.

Ballard Chris and Glenn Banks, 2003. Resource Wars: The Anthropology of Mining, Annual Review of Anthropology 32, pp. 287313.

-, 2009. Between a rock and a hard place: corporate strategy at the Freeport mine in Papua, 2001-2006?, in B. Resosudarmo and F. Jotzo (eds), Working with Nature against Poverty: Development, Resources and the Environment in Eastern Indonesia, Singapore, ISEAS, pp. 147-177.

Banaré Eddy, 2012. Les récits du nickel en Nouvelle-Calédonie (1853-1860), Paris, Honoré Champion, Francophonies.

—, 2014. Aspects littéraires de la recherche archivistique en Nouvelle-Calédonie: d'où vient le pionnier?, in V. Fillol et P.-Y. Le Meur (éds), Terrains océaniens : enjeux et méthodes, Paris, L'Harmattan, cahiers du Pacifique Sud, pp. 307-324.

Banks Glenn and Chris Ballard (eds), 1997. The Ok Tedi Settlement: issues, outcomes and implications, Canberra, ANU, Resource Management in Asia-Pacific, National Centre for Development Studies, Policy Paper n 27.

Barbançon Louis-José, 1992. Le Pays du nondit. Regards sur la Nouvelle-Calédonie, La Motte Achard, à compte d'auteur.

Bayard Pierre, 2009. Le plagiat par anticipation, Paris, Éditions de Minuit.

Bebbington Anthony, Leonith Hinojosa, Denise Humphreys BebBington, Maria Luisa Burneo and Ximena WarnaArs, 2008. Contention and Ambiguity: Mining and the Possibilities of Development, Development and Change 39 (6), pp. 887-914.

Benvivengo Yann, 2012. Immigration japonaise en Nouvelle-Calédonie: une illustration de l'affirmation du Japon dans le Pacifique, Journal de la Société des Océanistes 135 : Relations internationales et régionales en Océanie (S. Mohamed-Gaillard éd.), pp. 215-228.

Biersack Aletta, 1999. The Mount Kare python and his gold, American Anthropologist 101, pp. 68-87.

Bremmer Ian and Robert Joohnston, 2009. The rise and fall of resource nationalism, Survival 51(2), pp. 149-158. 
Brenneis Donald and Fred Myers (eds), 1984. Dangerous Words: Language and Politics in the Pacific, New York, New York University Press

Brutti Lorenzo, 2007. From fertility rituals to mining companies: Ecocultural issues and land rights in Oksapmin, Journal de la Société des Océanistes 125, pp. 249-255.

Burke Peter (ed.), 1991. New Perspectives on Historical Writing, Londres, Polity Press.

Callon Michel, 1981. Pour une sociologie des controverses techniques, Fundamentae Scientae 2, pp. 381-399.

Clifford James, 2013. Returns. Becoming Indigenous in the Twenty-First Century, Cambridge, Harvard University Press.

Clifford James and Georges Marcus (eds), 1986. Writing Culture. The Poetics and Politics of Ethnography, Berkeley, University of California Press.

Cooper Frederick, 1997. Modernizing Bureaucrats, Backward Africans, and the Development Concept, in F. Cooper and R. Packard (eds), International Development and the Social Sciences. Essays on the History and Politics of Knowledge, Berkeley, University of California Press, pp. 64-92.

Coumans Catherine, 2011. Occupying Spaces Created by Conflict: Anthropologists, Development NGOs, Responsible Investment, and Mining (with CA comment by S. Kirsch), Current Anthropology 52 (S3), pp. S29-S43.

Crook Tony, 2007. 'If you don't believe our story, at least give us half of the money': claiming ownership of the Ok Tedi Mine, PNG, Journal de la Société des Océanistes 125, pp. 221-228.

DASWHOOD Hevina, 2013. The Rise of Global Corporate Social Responibility. Mining and the Spread of Global Norms, Cambridge, Cambridge University Press.

Davies Charlotte Aull, 1999. Reflexive Ethnography. A Guide to Researching Selves and Others, Londres, Routledge.

Dawson Andrew, Jenny Hockey and Allison James (eds), 1997. After Writing Culture: Epistemology and Praxis in Contemporary Anthropology, Londres, Routledge, ASA Monographs.

Demmer Christine, 2007. Autochtonie, nickel et environnement. Une nouvelle stratégie kanak, Vacarme 39, pp. 43-48.

Esposito Roberto, 2000. Communitas. The Origin and Destiny of Community, Stanford, Stanford University Press.
Evans Julie, Ann Genovese, Alexander Reilly and Patrick Wolfe (eds), 2013. Sovereignty. Frontiers of Possibility, Honolulu, University of Hawai'i Press.

Fabian Johannes 2006 (éd. or. 1983). Le temps et les autres. Comment l'anthropologue construit son objet, préface d'Alban Bensa, Toulouse, Anarchasis.

-, 2006. The other revisited: Critical afterthoughts, Anthropological Theory 6 (2), pp. 139-152.

Fabiani Jean-Louis, 1997. Controverses scientifiques, controverses philosophiques. Figures, positions, objets, Enquête 5, pp. 11-34.

Fanon Frantz, 2011. Euvres, Paris, La Découverte.

FILER Colin, 1990. The Bougainville Rebellion, the Mining Industry and the Process of Social Disintegration in Papua New Guinea. Canberra Anthropology 13 (1), pp. 1-39.

- 2006. Custom, Law and Ideology in Papua New Guinea, The Asia Pacific Journal of Anthropology 7 (1), pp. 65-84.

- 2012, The development forum in Papua New Guinea: evaluating outcomes for local communities, in Marcia Langton and Judy Longbottom (eds), Community Futures, Legal Architecture: Foundations for Indigenous People in the Global Mining Boom, London, Routledge, pp. 45-58.

Filer Colin (ed.), 1999. Dilemmas of Development: The social and economic impact of the Porgera gold mine, 1989-1994, Canberra, Asia-Pacific Press.

Filer Colin, John Burton and Glenn Banks, 2008. The fragmentation of responsibilities in the Melanesian mining sector, in S. Ali and C. O'Faircheallaigh (eds), Earth Matters. Indigenous peoples, the extractive industry and corporate social responsibility, Sheffield, Greenleaf Publishing, pp. 163-179.

Filer Colin, Pierre-Yves Le Meur and JeanMichel Sourisseau (eds), à paraître. Local politics and large-scale mining. Perspectives from New Caledonia and Papua New Guinea, Canberra, ANU Epress.

Foster Robert J. (ed.), 1995. Nation Making. Emergent Identities in Postcolonial Melanesia, Ann Arbor, The University of Michigan Press.

-, 2002. Materializing the Nation. Commodities, Consumption, and Media in Papua New Guinea, Bloomington, Indiana University Press. 
Fuentes Carlos, 1979 (éd. or. mexicaine 1975). Terra Nostra, Paris, Gallimard.

Genette Gérard, 2007. Discours du récit. Essai de méthode (volume regroupant Figure III : Discours du récit [1972] et Nouveau discours du récit [1983]), Paris, Seuil.

Graff Stéphanie, 2013. Quand combat et revendication kanak ou politique de l'État français manient indépendance, décolonisation, autodétermination et autochtonie en Nouvelle-Calédonie, Journal de la Société des Océanistes 134, pp. 61-83.

Golub Alex, 2007. From Agency to Agents: Forging Landowner Identities in Porgera, in J. F. Weiner and K. Glaskin (eds), Customary Land Tenure and Registration in Australia and Papua New Guinea: Anthropological Perspectives, Canberra, ANU Epress, pp. 57-72.

GräTz Tilo, 2004. Les frontières de l'orpaillage en Afrique occidentale, Autrepart 30, cahier thématique Gouverner les hommes et les ressources. Dynamiques de la frontière interne, (J.-P. Chauveau, J.-P. Jacob et P.-Y. Le Meur eds), pp. 135-150.

Greimas Algirdas Julien et Joseph Courtès, 1993. Sémiotique. Dictionnaire raisonné de la théorie du langage, Paris, Hachette.

Grochain Sonia, 2013. Les dynamiques sociétales du projet Koniambo, Nouméa, Éditions IAC, Études et Synthèse.

Grochain Sonia, David Poithily et Jean-Michel Sourisseau, à paraître. From Anticipation to Practice: Social and Economic Management Put to the Test in the Metals Sector. An Example in New Caledonia's North Province, in C. Filer, P.-Y. Le Meur and J.-M. Sourisseau (eds), Local Politics and LargeScale Mining. Perspectives from New Caledonia and Papua New Guinea, Canberra, ANU Epress.

Hartog François, 2003. Régimes d'historicité. Présentisme et expérience du temps, Paris, Seuil.

—, 2005. Évidence de l'histoire. Ce que voient les historiens, Paris, Seuil.

Hau'ofa Epeli, 1993. Our Sea of Island, in E. Waddell, V. Naidu and E. Hau' ofa (eds), New Oceania: Rediscovering our Sea of Island, Fiji, University of the South Pacific, School of Social and Economic Development, pp. 2-19.

Hentsch Thierry, 2005. Le temps aboli : l'Occident et ses grands récits, Montréal, Presses de l'Université de Montréal/Bréal.

Hirschman Albert O., 1991. Deux siècles de rhétorique réactionnaire, Paris, Fayard.
Horowitz Leah S., 2003. La micropolitique de la mine en Nouvelle-Calédonie, Analyse des conflits autour d'un projet minier au sein d'une communauté kanak, Journal de la Société des Océanistes 117 : Nouvelle-Calédonie, 150 ans après la prise de possession (I. Leblic éd.), pp. 254-271.

-, 2012. Translation Alignment: ActorNetwork Theory and the power dynamics of environmental protest alliances in New Caledonia, Antipode 44 (3), pp. 806-827.

Hyndman David, 2005. Shifting Ecological Imaginaries in the Ok Tedi Mining Crisis in Papua New Guinea, Journal de la Société des Océanistes 120-121: Ethnoécologie en Océanie (F. Brunois éd.), pp. 76-93.

Imbun Benedict, 2011. Anthropology of Mining in Papua New Guinea Greenfields, New York, Nova Publishers.

Jorgensen Dan, 1990. Placing the past and moving the present: Myth and contemporary history in Telefolmin, Culture 10, pp. 47-56.

-, 2004. Who and what is a landowner? Mythology and marking the ground in a Papua New Guinea mining project, in Alan Rumsey and James Weiner (eds), Mining and Indigenous Lifeworlds in Australia and Papua New Guinea, Wantage, Sean Kingston Publishing, pp. 68-100.

Joyce Richard, 2013. Competing Sovereignties, London, Routledge.

KIrsch Stuart, 2006. Reverse Anthropology: Indigenous analysis of social and environmental relations in New Guinea, Stanford, Stanford University Press.

Latour Bruno, 1989. La science en action, Paris, Seuil.

-, 2002. La fabrique du droit. Une ethnographie $d u$ Conseil d'État, Paris, La Découverte.

-, 2006. Changer la société - Refaire de la sociologie, Paris, La Découverte.

LeBLic Isabelle, 1993. Les Kanak face au développement. La voie étroite, Grenoble, PUG.

-, 2007. Kanak Identity, New Citizenship Building and Reconciliation, Journal de la Société des Océanistes 125, pp. 271-282.

Leith Denise, 2003. The Politics of Power: Freeport in Suharto's Indonesia, Honolulu, University of Hawai'i Press.

Le Meur Pierre-Yves, 2012 (6-8 nov.). The New Caledonia/France relationship through the lens of the mine. Colonization and globalization, old and new, Pacific History Association 
Conference 2012 Generations: History in the Pacific, Histories in the Future, Panel "Revisiting the "French Pacific": connected or disconnected histories?», Wellington (NZ).

—, 2014. Anthropologie de la mine: travailler sur/dans/avec le secteur minier en NouvelleCalédonie, in V. Fillol et P.-Y. Le Meur (éds), Terrains océaniens : enjeux et méthodes, Paris, L'Harmattan, Cahiers du Pacifique Sud, pp. 175-194.

- à paraître. Conflict and agreement. The politics of Nickel in Thio, New Caledonia, in C. Filer, P.-Y. Le Meur and J.-M. Sourisseau (eds), Local Politics and Large-Scale Mining. Perspectives from New Caledonia and Papua New Guinea, Canberra, ANU Epress.

Le Meur Pierre-Yves, Chris Ballard, Glenn Banks and Jean-Michel Sourisseau, 2013. Two islands, four states: Comparing resource governance regimes in the Southwest Pacific, in J. Wiertz (ed.), Proceedings of the $2^{\text {nd }}$ International Conference on Social Responsibility in Mining (SRMining 2013, Santiago, Chile), University of Queensland, GECAMIN-SMI, pp. 191-199.

Le Meur Pierre-Yves, Leah Horowitz and Thierry Mennesson, 2013. «Horizontal» and «vertical» diffusion: the cumulative influence of Impact and Benefit Agreements (IBAS) on mining policy-production in New Caledonia, Resources Policy 38, pp. 648-656.

LeTté Michel, 2009. Débordements industriels dans la cité et histoire de leurs conflits aux $\mathrm{XIX}^{\mathrm{e}}$ et $\mathrm{XX}^{\mathrm{e}}$ siècles, Documents pour l'histoire des techniques 17, pp. 163-173.

LEVACHER Claire, à paraître. Indigenous people rights and the Goro-Nickel mining project in New Caledonia, in C. Filer, P.-Y. Le Meur, and J.-M. Sourisseau (eds), Local Politics and Large-Scale Mining. Perspectives from New Caledonia and Papua New Guinea, Canberra, ANU Epress.

LyOTARD Jean-François, 1979. La condition postmoderne. Rapport sur le savoir, Paris, Éditions de Minuit.

Masquelier Bertrand et Jean-Louis Siran (éds), 2000. Pour une anthropologie de l'interlocution. Rhétoriques du quotidien, Paris, L'Harmattan.

Mayes Warren and Nigel Chang, 2013. Cultural heritage management and local participation in the development of the Sepon mine, in J. Wiertz (ed.), Proceedings of the 2nd International Conference on Social Responsibility in Mining (SRMining 2013, Santiago, Chile),
University of Queensland, GECAMIN-SMI, pp. 55-62.

Mcintosh Ian, 2004. The iron furnace of Birrinydji, in Alan Rumsey and James Weiner (eds), Mining and Indigenous Lifeworlds in Australia and Papua New Guinea, Wantage, Sean Kingston Publishing, pp. 12-30.

Merlan Francesca, 1994. Narratives of Survival in the Post-Colonial North, Oceania 65, pp. 152-173.

Merle Isabelle, 1998. La construction d'un droit foncier colonial. De la propriété collective à la constitution des réserves en Nouvelle-Calédonie, Enquête 7, pp. 97-126.

Montalbetti Christine, 1998. Gérard Genette : une poétique ouverte, Paris, Bertrand-Lacoste.

Mosse David, 2004. Is Good Policy Unimplementable? Reflections on the Ethnography of Aid Policy and Practice, Development \& Change 35 (4), pp. 639-671.

Nakoro Elia, 2011 (21-25 Nov). Mining and Cultural Heritage Preservation in Fiji, International conference Mining in the Pacific: History, issues, perspectives, Noumea.

NasH June, 1993 (1 ${ }^{\text {e }}$ éd. 1979). We Eat the Mines and the Mines Eat Us. Dependency and Exploitation in Bolivian Tin Mines, New York, Columbia University Press.

O'Faircheallaich Ciaran, 2012. Curse or opportunity? Mineral revenues, rent-seeking and development in Aboriginal Australia, in Marcia Langton and Judy Longbottom (eds), Community Futures, Legal Architecture: Foundations for Indigenous People in the Global Mining Boom, London, Routledge, pp. 45-58.

PARRY Jonathan and Marc Bloch (eds), 1989. Money and the morality of exchange, Cambridge, Cambridge University Press.

Passeron Jean-Claude, 1991. Le raisonnement sociologique. L'espace non-poppérien du raisonnement naturel, Paris, Nathan.

Polier Nicole, 1996. Of mines and Min: modernity and its malcontents in Papua New Guinea, Ethnology 35, pp. 1-16.

Propp Vladimir, 1970 (1 éd. 1928). Morphologie du conte, Paris, Seuil.

Richer de Forges Bertrand et Michel Pascal, 2008. La Nouvelle-Calédonie, un "point chaud " de la biodiversité mondiale gravement menacé par l'exploitation minière, Journal de la Société des Océanistes 126-127: Spécial Environnement dans le Pacifique (J. Trichet et I. Leblic éds), pp. 95-112. 
Ricceur Paul, 1983. Temps et récit, vol. 1 : L'intrigue et le récit historique, Paris, Seuil.

—, 1984. Temps et récit, vol. 2 : La configuration dans le récit de fiction, Paris, Seuil.

—, 1985. Temps et récit, vol. 3 : Le temps raconté, Paris, Seuil.

—, 1990. Soi-même comme un autre, Paris, Seuil.

Robinson James, Ragnar Torvik and Thierry Verdier, 2006. Political foundations of the resource curse, Journal of Development Economics 79, pp. 447-468.

Roe Emery, 1994. Narrative Policy Analysis. Theory and Practice, Durham-London, Duke University Press.

Rosser Andrew, 2006. The Political Economy of the Resource Curse: A Literature Survey, Brighton, Institute of Development Studies, Working Paper 268.

SAID Edward, 2000. Culture et impérialisme, Paris, Le Monde.

Sanjek Richard, 1990. The Ethnographic Present, Man (N.S.) 26, pp. 609-628.

SAussol Alain, 1979. L'Héritage. Essai sur le problème foncier mélanésien en Nouvelle-Calédonie, Paris, Société des Océanistes, Publication de la SdO 40.

Shipton Parker and Mitzi Goheen, 1992. Introduction. Understanding African Landholding: Power, Wealth and Meaning, Africa 62 (3), pp. 307-325.

Shlomowitz Ralph and Doug Munro, 1992. The Ocean Island (Banaba) and Nauru labour trade 1900-1940, Journal de la Société des Océanistes 94 (1), pp. 103-117.

Sidaway James, 2007. Enclave space: a new metageography of development?, Area 39 (3), pp. 331-339.
Sivaramakrishnan K., 2000. Crafting the Public Sphere in the Forests of West Bengal, American Ethnologist 27 (2), pp. 431-461.

Somers Margaret, 1994. The Narrative Constitution of Identity: A Relational and Network Approach, Theory and Society 23 (5), pp. 605-649.

Strathern Marilyn, 2009, Land: Intangible or Tangible Property?, in T. Chesters (ed.), Land Rights. The Oxford Amnesty Lectures 2005, Oxford, Oxford University Press, pp. 13-38.

Sultan Patrick, 2011. La scène littéraire postcoloniale, Paris, Le Manuscrit, L’Esprit des Lettres.

Taussig Michael, 2010 (1 ${ }^{\mathrm{e}}$ éd. 1980). The Devil and Commodity Fetishism in South America, Chapel Hill, The University of North Carolina Press.

Thompson Anne-Gabrielle, 2000. John Higginson : un spéculateur-aventurier à l'assaut du Pacifique (Nouvelle-Calédonie, Nouvelles-Hébrides), Paris, L’Harmattan.

Veyne Paul, 1978. Comment on écrit l'histoire, Paris, Seuil.

WARD Halina, 2009. Resource nationalism and sustainable development: a primer and key issues, London, IIED, Working Paper.

White Geoffrey and Karen Ann Watson GeGEO (eds), 1990. Disentangling. Conflict Discourse in Pacific Societies, Stanford, Stanford University Press.

White Hayden, 2009 (éd. or. américaine 1973). Poétiques de l'histoire, Labyrinthe 33 (2), pp. 21-65.

-, 1973. Metahistory: The Historical Imagination in Nineteenth-Century Europe, Baltimore, John Hopkins University Press.

- 1990. The Content of the Form: Narrative Discourse and Historical Representation, Baltimore, John Hopkins University Press. 